IOS Press

\title{
The role of lipocalin-2 serum levels in the diagnostics of endometrial cancer
}

\author{
Aneta Cymbaluk-Płoska ${ }^{\mathrm{a}, *}$, Anita Chudecka-Głaz ${ }^{\mathrm{a}}$, Ewa Pius-Sadowska ${ }^{\mathrm{c}}$, Bogusław Machaliński $^{\mathrm{c}}$, \\ Agnieszka Sompolska-Rzechuła $^{\mathrm{d}}$, Sebastian Kwiatkowski ${ }^{\mathrm{b}}$ and Janusz Menkiszak ${ }^{\mathrm{a}}$ \\ ${ }^{a}$ Department of Gynecological Surgery and Gynecological Oncology of Adults and Adolescents, Pomeranian \\ Medical University, Szczecin, Poland \\ ${ }^{\mathrm{b}}$ Department of Obstetrics and Gynecology, Pomeranian Medical University, Szczecin, Poland \\ ${ }^{\mathrm{c}}$ General Pathology Department, Pomeranian Medical University, Szczecin, Poland \\ ${ }^{\mathrm{d}}$ West Pomeranian University of Technology, Szczecin, Poland
}

\begin{abstract}
.
BACKGROUND: Endometrial cancer is one of the most common tumor of the woman genital organs.

OBJECTIVE: The goal of this study was to determine the lipocalin-2 levels in patients with endometrial cancer compared to those with normal endometrium or mild endometrial pathologies.

METHODS: Study included 123 patients with BMI $>21 \mathrm{~kg} / \mathrm{m}^{2}$ who were admitted due to abnormal bleeding, in which 52 patients with endometrial cancer. The NGAL, CA125, HE4 serum levels were determined for all patients.

RESULTS: Significantly lower median NGAL serum levels were found in a group of patients with normal endometrium compared to the endometrial cancer group, $p=0.006$. NGAL protein area under ROC curves value as a diagnostic test, differentiating between endometrial cancer and other benign changes endometrium is AUC $-0.81(p<0.00001)$. The NGAL protein had a high sensitivity in all patients included in the analysis: $84 \%$ vs. $82 \%$ in pre-menopausal patients, and $81 \%$ in postmenopausal women with a specificity of $78 \%, 80 \%$ and $87 \%$, respectively. The independent variable for FIGO and model logistic regression proves that NGAL is statistically significant $(p=0.000602)$, the odds ratio is 3.66 . The model for grading shows, that NGAL increase by one $\mathrm{ng} / \mathrm{ml}$ increases risk chances by 2.32 times in diagnosis with less cancer differentiation.

CONCLUSIONS: Our preliminary studies demonstrate that lipocalin-2 may be of value in the diagnostics of uterine body cancers.
\end{abstract}

Keywords: Lipocalin-2, CA125, HE4, benign endometrial changes, endometrial cancer

\section{Introduction}

Endometrial cancer is widely prevalent, especially in the developed countries. Obesity is the main factor influencing its development. Adipose tissue is involved in metabolism, immune response and plays an endocrine function. In 2007 adipose tissue was shown to produce a glycoprotein - neutrophil gelatinaseassociated lipocalin (NGAL), also known as sidero-

\footnotetext{
* Corresponding author: Aneta Cymbaluk-Płoska, Department of Gynecological Surgery and Gynecological Oncology of Adults and Adolescents, Pomeranian Medical University of Szczecin, Al. Powstańców Wielkopolskich 72, 70-111 Szczecin, Poland. E-mail: anetac@data.pl.
}

calin or lipocalin-2 [30]. It was first identified in active neutrophils as a protein covalently bound to human neutrophil gelatinase, hence the name. This 178-amino acid protein belongs to the family of extracellular matrix proteins. It is a risk factor for insulin resistance, diabetes, and chronic or acute inflammatory conditions $[38,41,44]$. It also participates in oncogenesis $[9,10,20]$.

Elevated expression of lipocalin-2 was demonstrated in tissues obtained from tumors of the breast, colon, ovary or pancreas $[11,17,22,29,39,40,42,43,45]$. Reduced expression of E-cadherin and resultant increased mobility and invasiveness of tumor cells was noted in colorectal cancer cell lines overexpressing lipocalin2 [17]. Recent studies on endometrial cancer showed

ISSN 1574-0153/19/\$35.00 (C) 2019 - IOS Press and the authors. All rights reserved

This article is published online with Open Access and distributed under the terms of the Creative Commons Attribution Non-Commercial License (CC BY-NC 4.0). 
that lipocalin-2 contributes to progression of this tumor. Immunohistochemical studies confirmed greater expression of lipocalin-2 in endometrial cancer compared to atypical endometrial hyperplasia [21,27,28]. The goal of this study was to evaluate NGAL diagnostic value in diagnosing endometrial cancer in patients with abnormal bleeding and pathological endometrium changes in the USG image.

\section{Material and methods}

Study included 123 patients with BMI $>21 \mathrm{~kg} / \mathrm{m}^{2}$ who were admitted due to abnormal bleeding from the genitals. Patients eligible for the study were preand post-menopausal with abnormal bleeding from the uterine cavity and with changes in the ultrasound image. USG TV was performed for all patients, evaluating the endometrium of the uterine cavity. All patients signed informed consent to participate in the study. The study protocol was approved by the PUM Ethical Committee. USG TV was performed for all patients, evaluating the mucous membrane of the uterine cavity. Four women with high baseline lipocalin-2 levels $(>2000 \mathrm{ng} / \mathrm{mL}$ ) were excluded from the study. All subjects had elevated creatinine levels and were under nephrological care due to CKD - chronic kidney disease. Material for histopathological examination was taken during procedures like: abrasion, hysteroscopy and radical surgery in case of confirmed endometrial cancer. Following surgical treatment and the results of histopathological examination patients were assorted into four groups (Table 1):

- Patients with endometrial cancer $-n=52$

- Patients with normal endometrium $-n=30$

- Patients with endometrial polyps $-n=20$

- Patients with submucosal leiomyomas $-n=17$

In each of the subgroups patients were considered for hormonal status and depending on the BMI. BMI was calculated using following formula:

$$
B M I=\frac{\text { weight }(\mathrm{kg})}{\text { height }\left(\mathrm{m}^{2}\right)}
$$

Among the group of patients with endometrial cancers we identified 47 patients with endometrioid endometrial carcinoma, and 5 patients with nonendometrioid endometrial carcinoma.

Patients from the endometrial cancer group were divided according to tumor grading into $\mathrm{G} 1=18, \mathrm{G} 2=$ 24 and G3 = 10 subgroups, as well as depending on clinical tumor staging:
Table 1

Population data characteristics of patients from control and examined group

\begin{tabular}{clc}
\hline Group & \multicolumn{1}{c}{ Characteristic } & Patients \\
\hline Control & Mean age & 53 years \\
group & Benign pathology & 67 \\
$n=67$ & Normal endometrium & 30 \\
& Submucosal leiomyomas & 17 \\
& Endometrial polyps & 20 \\
Examined & Mean age & 58 \\
group & Grading & 52 \\
$n=52$ & G1 & 18 \\
& G2 & 24 \\
& G3 & 10 \\
& FIGO & 52 \\
& I + II & 42 \\
& III + IV & 10 \\
& Histopathology & \\
& Endometrioid endometrial carcinoma & 47 \\
& Non-endometrioid endometrial carcinoma & 5 \\
& Papilary serosus carcinoma & 3 \\
& Clear cell carcinoma & 2 \\
\hline
\end{tabular}

- FIGO 1 and 2 patients, $n=42$,

- FIGO 3 and 4 patients, $n=10$.

Five milliliters of blood was collected from each patient for protein levels determination on the occasion of routine preoperative testing and centrifuged. The serum was subsequently frozen and stored at $-70^{\circ} \mathrm{C}$.

\subsection{Assay analysis}

Lipocalin-2/NGAL concentrations were quantified in serum by fluorescent bead-based immunoassays (Luminex Corporation, Austin, TX, USA) using commercial Human Cardiovascular Disease (CVD) Magnetic Bead Panel 2 (Merck Millipore, Billerica, MA, USA). CA125 was determined using ARCHITECT CA 125 II assay [Reagent Kit No 2K45], Abbott Diagnostics, Illinois, USA. Analytical sensitivity was $1.0 \mathrm{U} / \mathrm{mL}$. Serum HE4 concentrations were measured using the Elecsys ECLIA assay [Kit No 05950929190], Roche Diagnostics Ltd, Rotkreuz, Switzerland running on the cobas e 601 analyzer. Detection limit $15.0 \mathrm{pmol} / \mathrm{L}$. Statistical analysis was performed using STATISTICA 9.1 PL software. Descriptive characteristics of the examined population of patients were prepared, including the minimum, maximum, mean and median values. Because the distributions of the study traits were not normal, for evaluation positional parameters such as median were used. Non-parametric tests with Kruskal-Wallis test and Dunn's post-hoc test for comparisons between three groups were used and Mann-Whitney's U-test for comparison between two 


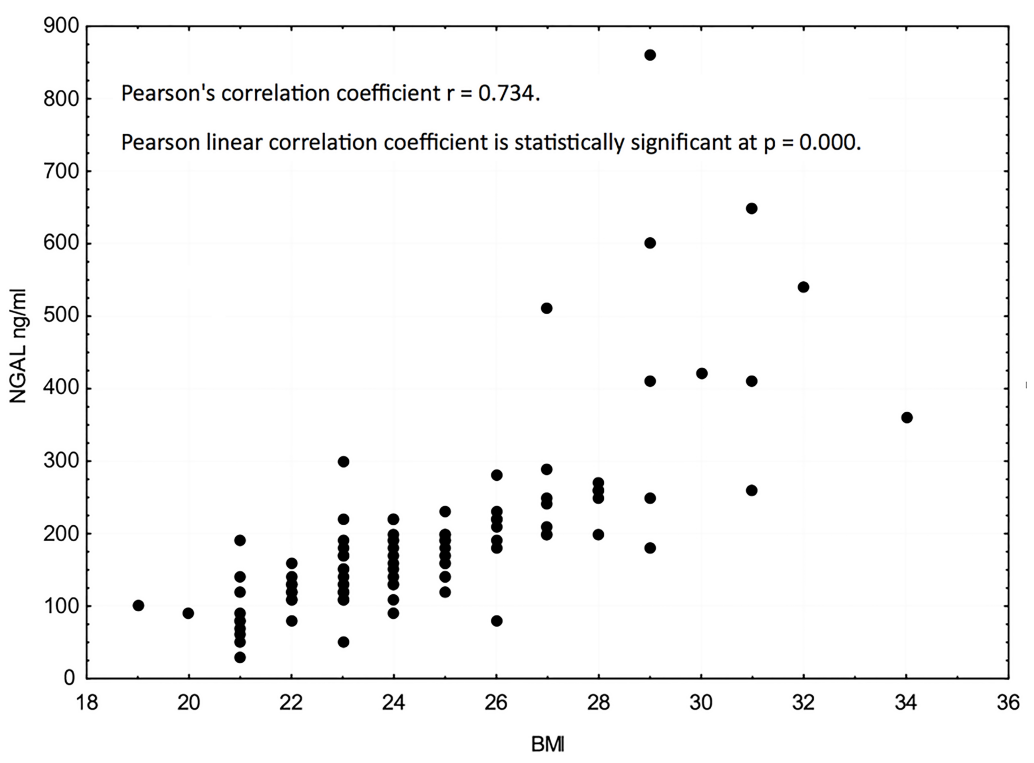

Fig. 1. The relationship between NGAL and BMI in the whole group of patients.

Table 2

Protein concentrations distribution in the individual subgroups

\begin{tabular}{|c|c|c|c|c|c|c|}
\hline & $n$ person & Mean & Median & Min & Max & SD \\
\hline \multicolumn{7}{|c|}{ Endometrial cancer } \\
\hline Age & 52 & 57.75 & 54.00 & 40.00 & 82.00 & 9.89 \\
\hline $\mathrm{NGAL}[\mathrm{ng} / \mathrm{ml}]$ & 52 & 198 & & 110 & 460 & 120 \\
\hline HE4 [p & 52 & 88.34 & 90.12 & 54.6 & 129.0 & 10.23 \\
\hline & 52 & 36.3 & & 22.6 & 52.2 & 6.1 \\
\hline \multicolumn{7}{|c|}{ Normal endometrium } \\
\hline Age & 30 & 59.58 & 60.00 & 43.00 & 81.00 & 10.69 \\
\hline NGAl & 30 & 115 & 109 & 91 & 150 & 86 \\
\hline $\mathrm{HE} 4$ & 30 & 54.84 & 55 & 47.32 & 63.4 & 7.8 \\
\hline CA1 & 30 & 22.76 & 23.91 & 18.11 & 48.3 & 5.32 \\
\hline \multicolumn{7}{|c|}{ Endometrial polyp } \\
\hline Age & 20 & 54.40 & 52.50 & 41.00 & 70.00 & 8.73 \\
\hline & & 125 & & 50 & 250 & 63 \\
\hline HE4 & 20 & 52.33 & & 41.99 & 63.66 & 6.9 \\
\hline $\mathrm{CA} 125[\mathrm{U} / \mathrm{ml}]$ & 20 & 24.61 & 25.75 & 16.11 & 38.17 & 4.32 \\
\hline \multicolumn{7}{|c|}{ Submucosal leiomyoma } \\
\hline Age & 17 & 50.64 & 49.00 & 42.00 & 62.00 & 6.23 \\
\hline $\mathrm{NGAL}[\mathrm{ng} / \mathrm{ml}]$ & 17 & 155 & 145 & 30 & 300 & 54 \\
\hline HE4 [pmol/l] & 17 & 55.01 & & 47.21 & 69.2 & 7.23 \\
\hline $\mathrm{CA} 125[\mathrm{U} / \mathrm{ml}]$ & 17 & 23.84 & 24.89 & 19.33 & 31.22 & 3.99 \\
\hline
\end{tabular}

NGAL - neutrophil gelatinase associated lipocalin, HE4 - human epididymis protein 4 , CA125 - cancer antigen 125 , SD - standard deviation.

groups in terms of distribution compatibility of analyzed variables were used.

For the selected groups, the receiver operating characteristic (ROC) curves were obtained and the area under curve (AUC) was calculated with $95 \%$ confidence intervals according to the nonparametric method of DeLong [12]. A $p$ value of $<0.05$ was considered as statistically significant.
The study used a logit model, where $p$ - the probability of occurrence of a specific event $\mathrm{x}$ - the chance occurrence of a particular event. Unknown model parameters are estimated using generalized largest squares or maximum likelihood method. To interpret it is used odds ratio understood as the ratio of the probability that the event occurs to the probability that this case does not appear. When assessing the correctness of the model are counted correctly and incorrectly classified cases.

\subsection{Ethical approval}

Resolution number: KB-0012/77/12 of the Bioethics Committee of the Pomeranian University of Medicine in Szczecin of 13 October 2012. All procedures performed in studies involving human participants were in accordance with the ethical standards of the institutional and/or national research committee and with the 1964 Helsinki declaration and its later amendments or comparable ethical standards.

\subsection{Informed consent}

Written informed consent was provided by the patients as well as the physician. All the patients have signed informed consent for the study, which has been signed and initialed by the doctor on each side. Patients read carefully the information and were able to ask questions. 


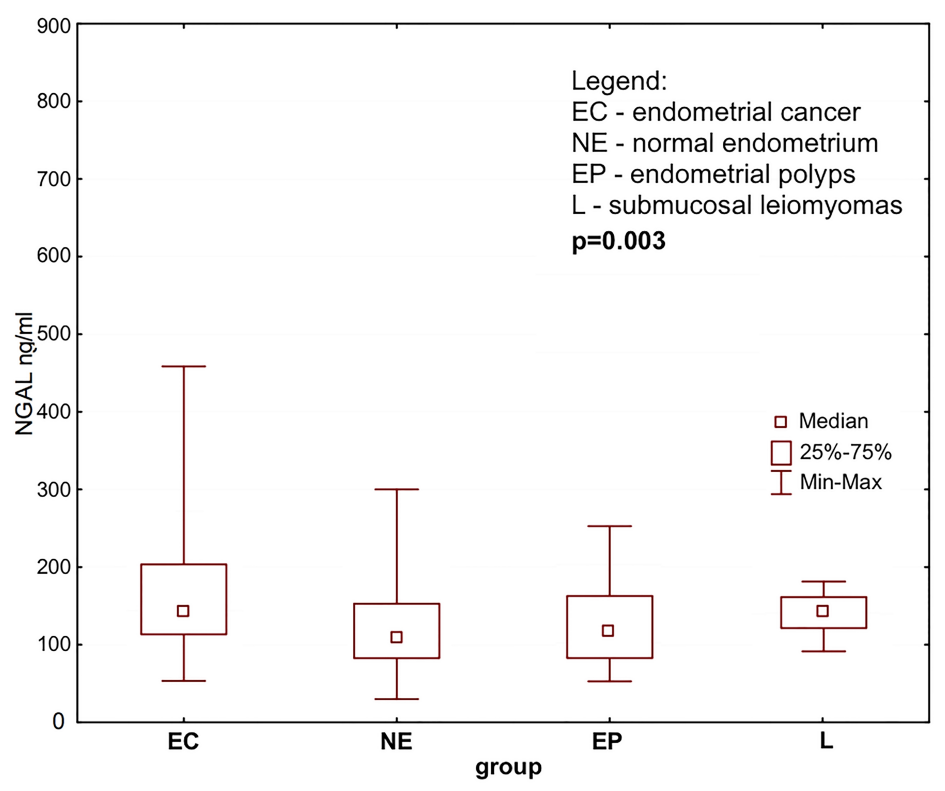

Fig. 2. The differences between the NGAL medians in groups.

\subsection{Consent for publication}

All patients included in the study signed conscious consent.

\section{Results}

The Kruskall-Wallis test show no differences in age distributions in the populations of the study groups. Statistically significant correlations were noted between patient BMI and concentrations of lipocalin-2 protein $(r=0.735 ; p \leqslant 0.001)$ in the whole group of patients. No statistically significant differences with regard to BMI values were noted among particular groups - Fig. 1 .

There was no correlation between patient BMI and serum concentrations of HE4 and CA125. We observed statistically significant differences in mean serum levels of lipocalin-2, HE4 and CA125 between the group of endometrial cancer patients and the group of patients with benign changes endometrium, both as regards the entire study population and after stratification based on BMI and menopausal status. The distribution of protein concentrations in the individual subgroups are shown in Table 2.

The distribution of lipocalin-2 was compared in patients group with normal endometrium and in group with endometrial cancer. A statistically significant difference was found between the distributions ( $p=$
0.006). Lipocalin-2 distributions in the polyp and leiomyoma patient group was compared with the endometrial cancer patients group and found that the distributions are significantly different $p=0.004, p=$ 0.03. A similar situation was observed during comparison of lipocalin-2 serum in leiomyomas patients and normal endometrium patients. Statistically significant differences were found in the analyzed distributions $(p=0.008)$. There were no statistically significant differences in the concentration of lipocalin- 2 between patients with endometrial polyps and those with normal endometrium $(p=0.39)$. Figure 2 illustrates results obtained with a K-W test and indicates significant differences between populations of the examined patient groups $(p=0.003)$.

\subsection{Analysis of ROC curves, assay sensitivity and specificity}

In order to evaluate the diagnostic values of lipocalin-2, HE4 and CA125, ROC curves were plotted and the areas under the ROC curves (AUC) were calculated. Area under ROC curves for lipocalin-2 protein as a diagnostic test, differentiating between endometrial cancer and other benign changes endometrium is AUC - $0.81(p<0.00001)$. Area under ROC curves for lipocalin-2 protein as a diagnostic test, differentiating between endometrial cancer and normal endometrium is AUC - $0.76(p=0.00003)-$ Fig. 3. On the other hand, the area under the curve for differen- 
Table 3

Protein concentration behavior depending on the clinical stage and cancer histopathological differentiation

\begin{tabular}{clccccc}
\hline & & endometrioid/non-endometrioid & G1/G2 & G2/G3 & G1/G3 & FIGO I, II/FIGO III, IV \\
\hline NGAL [ng/ml] & mean & $195 / 220$ & $167 / 174$ & $174 / 280$ & $167 / 280$ & $170 / 240$ \\
& median & $186 / 223$ & $170 / 181$ & $181 / 297$ & $170 / 297$ & 0.003 \\
& $p$ & $\mathrm{NS}$ & $\mathrm{NS}$ & $\mathrm{NS}$ & 0.0027 \\
$\mathrm{HE} 4[\mathrm{pmol} / \mathrm{l}]$ & mean & $88.6 / 93.2$ & $84.2 / 88.8$ & $88.8 / 102.2$ & $84.2 / 102.2$ & $74.5 / 104.3$ \\
& median & $88.0 / 92.8$ & $85.0 / 88.2$ & $88.2 / 101.3$ & $85.0 / 101.3$ & $77.3 / 106.2$ \\
$\mathrm{CA1} 25[\mathrm{U} / \mathrm{ml}]$ & $p$ & $\mathrm{NS}$ & $\mathrm{NS}$ & $\mathrm{NS}$ & 0.02 & 0.03 \\
& mean & $32.1 / 26.7$ & $31.8 / 39.2$ & $39.2 / 36.5$ & $31.8 / 36.5$ & $26.9 / 43.7$ \\
& median & $33.0 / 27.4$ & $32.5 / 37.3$ & $37.3 / 36.3$ & $37.3 / 36.3$ & $27.4 / 44.5$ \\
& $p$ & $\mathrm{NS}$ & $\mathrm{NS}$ & $\mathrm{NS}$ & $\mathrm{NS}$ & 0.003 \\
\hline
\end{tabular}

NGAL - neutrophil gelatinase associated lipocalin, HE4 - human epididymis protein 4, CA125 - cancer antigen 125, FIGO - Fédération Internationale de Gynécologie Obstétrique (International Federation of Gynecology and Obstetrics), G [1-3] - grading, $p$ - calculation of statistical significance with the help of Mann-Whitney's U-test.
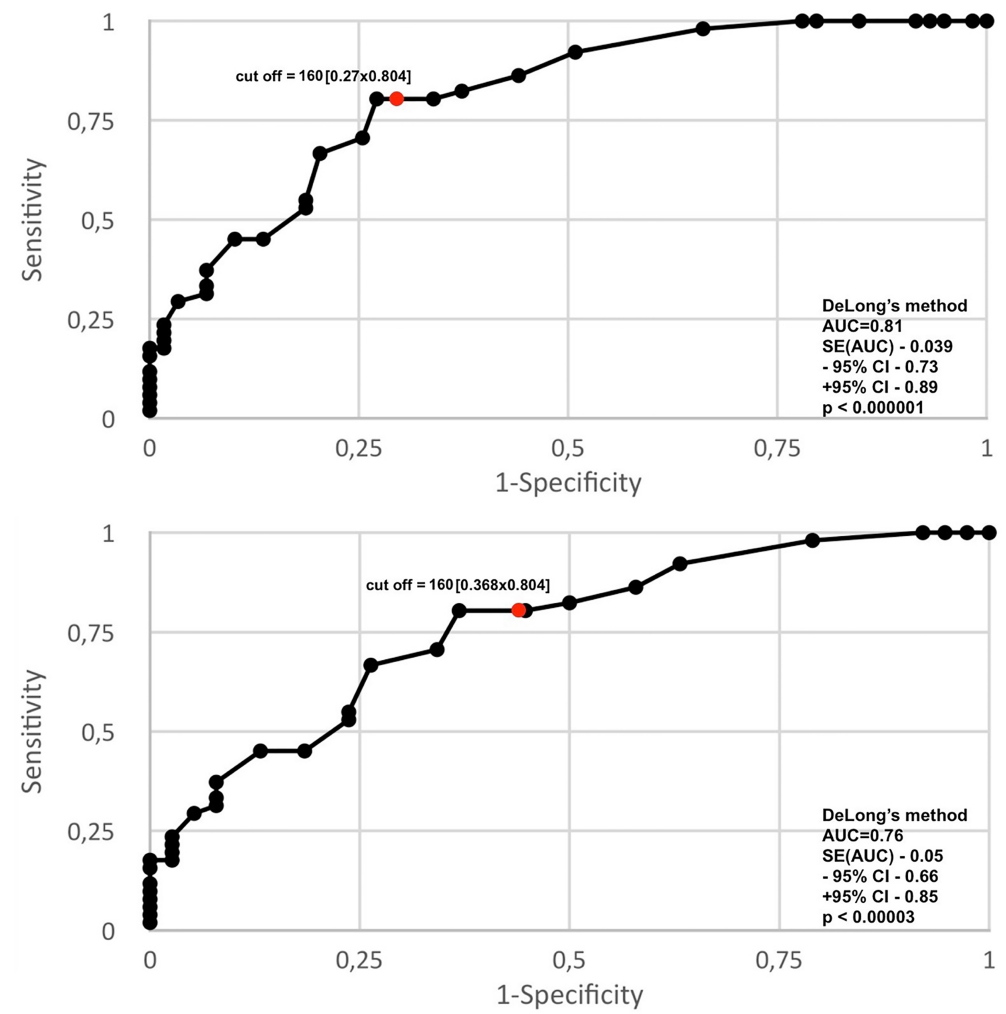

Fig. 3. NGAL ROC curve of endometrial cancers and endometrium benign changes comparison with NGAL ROC curve of endometrium cancers and normal endometrial.

tiation between endometrial cancer and leiomyomas is amounted to AUC -0.74 for $p=0.0033$. For HE4 and CA125 the AUC values were 0.74 and 0.53 respectively - Fig. 4, for differentiating between endometrial cancer and other benign endometrial lesions, values 0.80 and 0.61 ( $p=0.0004 / p=0.05)$ for differential diagnosis between endometrial cancer patients and normal endometrium and 0.76 and 0.66 for endometrial cancer patients and leiomyomas $(p=0.01 / p=$ 0.04).
Table 4 shows the sensitivity and specificity values for lipocalin-2, HE4, and CA125 based on hormonal status. We found higher sensitivity of lipocalin2 protein for all analyzed women (84\%) and for premenopausal and postmenopausal women $(82 \% / 81 \%)$ compared to the HE4 marker $(66 \%$ and $64 \% / 67 \%$ respectively) and CA125 (52\%, 63\% and 54\%). The HE4 marker showed a greater specificity compared to lipocalin-2 and CA125 in two study group and was: $93 \%$ vs $78 \%$ vs. $54 \%$ among all analyzed women, $90 \%$ 
HE4

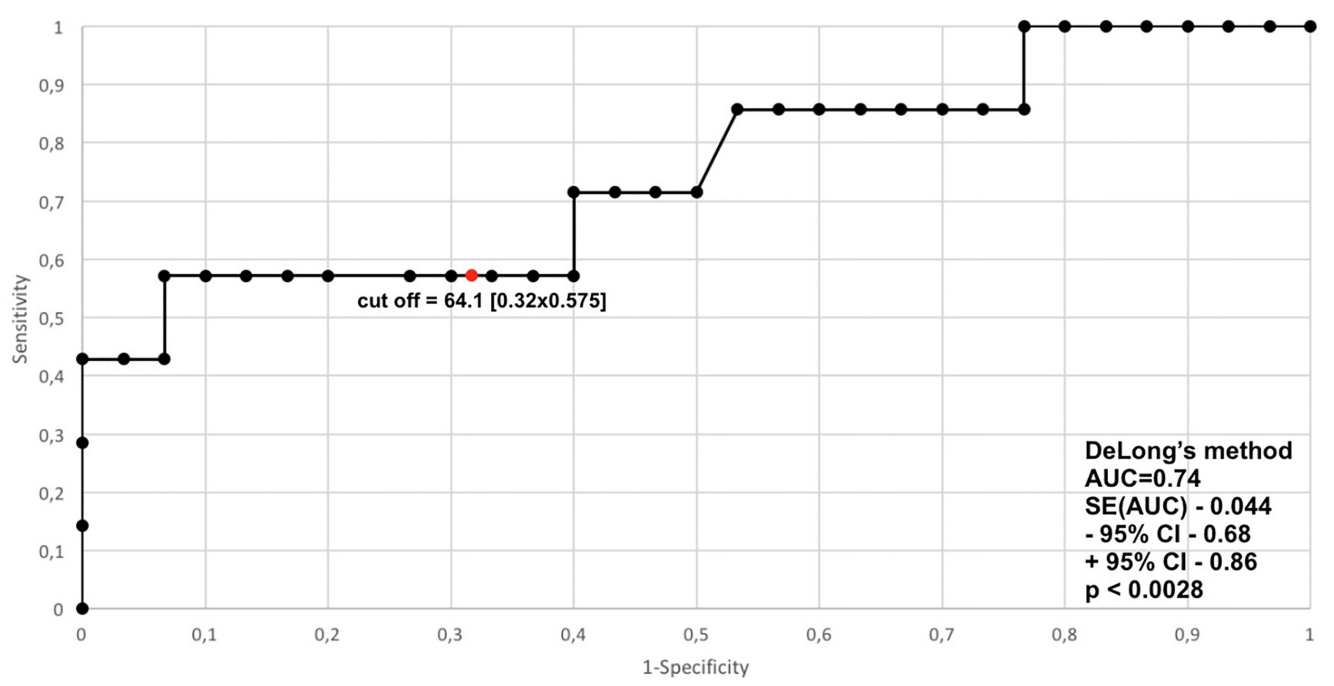

CA125

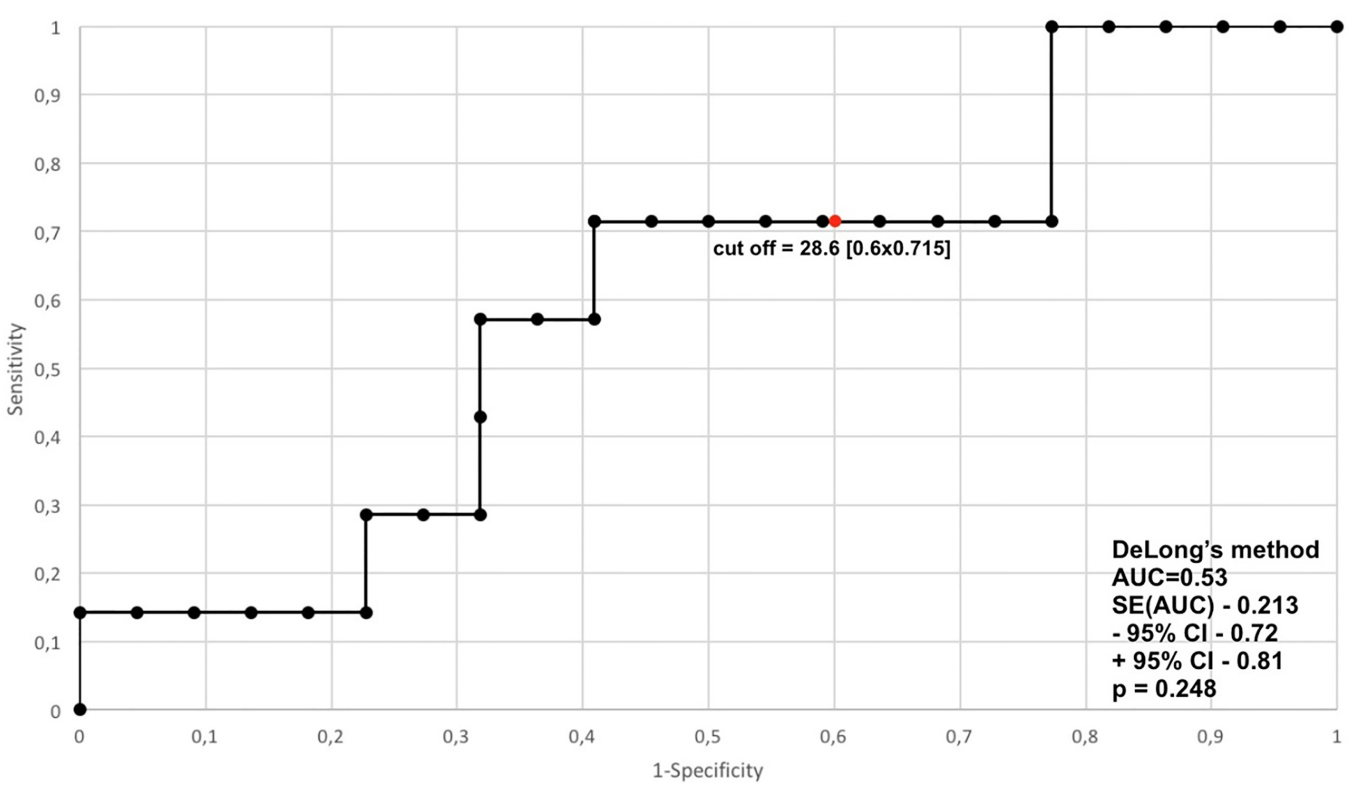

Fig. 4. HE4 and CA125 ROC curves for endometrial cancers and endometrium benign changes.

vs $80 \%$ vs $59 \%$ in premenopausal women and $85 \%$ vs $87 \%$ vs. $64 \%$ in the postmenopausal group.

\subsection{Lipocalin-2 protein concentrations analysis depending on the degree of histopathological differentiation and clinical stage of the cancer}

We demonstrated statistically significant correlation between serum concentrations lipocalin-2 among patients with various degrees of histopathological tumor differentiation (G3 versus G1) $p=0.003$. Significantly higher statistical mean levels of lipocalin-2 protein (240 ng/mL) were noted among patients with higher clinical staging compared to patients with lower staging (170 ng/mL), $p=0.0027$ - Table 3 .

\subsection{FIGO and lipocalin-2 logistic regression model}

A logistic regression model for the lipocalin-2 protein was created regarding FIGO. The odds ratio is 3.66. The model created reveals that the chances of a patient being in a more advanced group is higher 
Table 4

Individual proteins sensitivity and specificity depending on hormonal status

\begin{tabular}{lccc}
\hline & NGAL & HE4 & CA125 \\
\hline sensitivity & $84 \%$ & $66 \%$ & $52 \%$ \\
specificity & $78 \%$ & $93 \%$ & $54 \%$ \\
sensitivity PM & $82 \%$ & $64 \%$ & $63 \%$ \\
specificity PM & $80 \%$ & $90 \%$ & $59 \%$ \\
sensitivity M & $81 \%$ & $67 \%$ & $54 \%$ \\
specificity M & $87 \%$ & $85 \%$ & $64 \%$ \\
\hline
\end{tabular}

NGAL - neutrophil gelatinase associated lipocalin, HE4 - human epididymis protein 4 , CA125 - cancer antigen 125 , PM - premenopausal, $\mathrm{M}$ - postmenopusal.

for increased level of lipocalin-2. The model is statistically significant, $p=0.000001$. The area under the curve is $\mathrm{AUC}=0.884(p=0.000003)$. For the grading model, the odds ratio is 2.32 , which means that with the increase of lipocalin-2 serum level, the risk of being diagnosed with less differentiated endometrial cancer increases. The model is statistically significant, $p=$ 0.00024 . The $\mathrm{AUC}=0.764$ and $p=0.00016$.

\section{Discussion}

In $80 \%$ of cases endometrial cancer is associated with overweight or obesity. Adipose tissue plays an endocrine role, excreting adipokines, which play various activities in the body. Lipocalin-2 plays a role in development of insulin resistance, glucose intolerance and chronic inflammation. Developing inflammatory process leads to increased production of proinflammatory cytokines [8].

There are similar reports in the literature saying that obese and overweight patients have higher concentrations of lipocalin-2 [30,38,41,44]. In obese patients the paracrine signaling loop becomes dysregulated. High concentrations of adipocytokines begin to interact, weakening the role of adipose tissue as an immune organ and leads to augmented cell migration and endothelial damage.

Endometrial actions of lipocalin-2 have not been fully investigated. In their study on endometrial cancer cell lines Lin et al. observed reduction in initiated apoptosis, change in cell proliferation and stimulation of cytokine secretion, especially interleukin 8 and 6, after 24 hours of incubation. It was shown in the above studies that secretion of lipocalin-2 also depends on sex hormones, particularly estrogens, which are related to proliferation and apoptosis periodically taking place in the endometrium of menstruating women. Elevated levels of lipocalin-2 are noted in patients with higher serum glucocorticoid and estrogen concentrations [23].
Our study revealed augmented lipocalin-2 levels in patients with leiomyomas compared to those with normal endometrium. Lipocalin-2 elevation may result from higher concentrations of estrogens among patients with leiomyomas. Lipocalin-2 is also considered an acute phase protein [18]. The peak of incidence of endometrial cancer is observed in the perimenopausal age, which is associated with relative hyperestrogenism and resultant increase in lipocalin2 expression. Moreover, lipocalin-2 production takes place in the adipose tissue. The greater the BMI, the higher serum lipocalin-2 concentration. Greater amounts of fat tissue are associated with more extensive aromatization of adrenal androstendione to estrone - the main type of estrogen in postmenopausal women.

Considering the carcinogenic effects of lipocalin-2 and its production in follicular tissue, we have decided to investigate the diagnostic potential of this protein as a marker in endometrial cancer.

We have shown that the cut-off point for serum concentration of lipocalin-2 that differentiates endometrial benign changes from endometrial cancer is $160 \mathrm{ng} / \mathrm{ml}$. The sensitivity of the test protein was greater than the sensitivity of the HE4 and CA125 markers in the whole patient population, as well as the hormone status of the patients. The sensitivity and specificity of the HE4 marker was significantly higher than CA125, which corresponds to Liu et al. reports. Comparing early detection opportunities of endometrial cancer with CA125 and HE4, they have shown that the HE4 marker is more sensitive and specific regardless of the age of the patients and their hormone status [25].

In our studies, the CA125 AUC was 0.53 and HE4 AUC was 0.74, which was comparable to the results presented by Angioli et al., where the AUC was 0.54 and 0.86 respectively [4]. Angioli in the summary review of 5 studies found that all authors found statistically significantly higher HE4 concentration in the group of patients with endometrial cancer compared to the control group. The ROC and AUC curves for HE4 are in range from 0.78 to 0.88 , which in each case contained the HE4 marker criteria for a good diagnostic test. Moreover, in studies of three independent authors, differences in HE4 serum levels were also statistically significant between the control group and the stage I group [3].

Two authors in their work suggest that in the case of CA125 marker, its serous level increases only in $10 \%$ of patients with early stage of endometrium cancer (I and II) [34,35]. Based on their studies, Angioli and 
Plotti et al. recommended creation of REM or refined REM B algorithm, according to HE4 marker level and endometrium thickness, which was intended to stratify patients to low or high endometrium cancer risk groups $[1,32,33]$.

The specificity of lipocalin-2 was slightly poorer than HE4 marker for the whole group of patients and premenopausal patients, whereas in the postmenopausal patients group, the lipocalin-2 specificity was higher than HE4 specificity and was respectively $87 \% / 85 \%$. The cut-off limit for HE4 between the benign endometrial lesions and endometrial carcinomas in our study was $64.1 \mathrm{pmol} / \mathrm{l}$ and was slightly higher than the cut-off point for HE4 in studies by Gasiorowska et al. (58.08 pmol/l) [15]. For CA125 maker cut-off was at level of $28.6 \mathrm{U} / \mathrm{ml}$, opposed to meta-analysis cut-offs from Patsner and Yim $(20 \mathrm{U} / \mathrm{ml})$ for early stage of endometrial carcinoma [31]. Hu et al. emphasizes the fact that the HE4 marker is more sensitive than CA125, but the results of these markers as diagnostic tests are not satisfactory [16].

Angioli et al. confirmed that the HE4 level over $70 \mathrm{pmol} / \mathrm{l}$ at diagnosis had $67 \%$ sensitivity and $53 \%$ specificity in predicting recurrences of endometrial cancer [2,5]. Lipocalin-2 concentration was analyzed in relation to tumor size, advancement and presence of metastases [24]. It was demonstrated that elevated lipocalin-2 expression in endometrial cancer is tightly correlated with greater tumor aggressiveness and poorer prognosis [26]. Increased lipocalin-2 expression was noted in endometrial cancers compared to atypical or simple endometrial hypertrophy [27].

Miyamoto et al. proved in their studies that high expression of lipocalin-2 and its receptor SLC22A17 is associated with poorer prognosis in a group of patients with endometrial cancer [28].

High level of lipocalin-2 expression is associated with elevated concentrations of IL-8, which is responsible for increased cell proliferation, inhibition of apoptosis and cell migration. Our studies showed that higher clinical staging of endometrial cancer correlates with increase of lipocalin-2 serum concentration. Concentration of lipocalin- 2 have been examined in other cancers as well. Observations regarding breast cancer demonstrate increasing lipocalin-2 expression with advancement of the disease [39]. Similar reports pertain to chronic myeloid leukemia [19,20]. Expansion of tumor mass with increasing lipocalin-2 expression has also been described [6]. Moniaux et al. reported that lipocalin-2 might be a marker for early detection of pancreatic cancer [29]. Patients with elevated levels of lipocalin-2 are characterized by worse prognosis in esophageal and stomach cancer, although some publications report protective function of lipocalin-2 in thyroid and renal cancer $[6,7,13,14,19,36,37,42]$.

The high sensitivity of lipocalin-2 with comparable specificity to HE4 allows us to believe that lipocalin2 will prove to be useful in the endometrium cancer diagnosis.

Lipocalin-2 as a protein that combines the features of a metabolic factor and proliferative factor has a multidirectional ability to participate in the development of endometrial cancer. Proliferative effects of lipocalin-2 are both direct and indirect, through interaction with extracellular matrix metalloproteinases.

In our research, the relationship between BMI and lipocalin-2 raw materials has been confirmed. The results obtained by us are consistent with the reports of other authors [38]. In a group of obese patients, the sensitivity of lipocalin- 2 protein differing endometrium benign changes from endometrium cancer may be slightly lower, but still meets the criteria of a good diagnostic test.

Studies on a larger group of patients will unambiguously answer question whether or not lipocalin-2 serum concentration will allow us to isolate patients with high risk of endometrial cancer and subject them for endometrial sampling.

\section{Conclusion}

Our preliminary studies demonstrate that lipocalin2 , which reaches significantly higher levels in patients with endometrial cancer, may be of value in the diagnostics of uterine body cancers.

\footnotetext{
Abbreviations

NGAL neutrophil gelatinase-associated lipocalin siderocalin or lipocalin-2

HE4 Human Epididymis Protein 4

CA125 cancer antigen 125

CKD chronic kidney disease

PM premenopausal status

M menopausal status

BMI body mass index

ROC receiver operating characteristic curves

AUC area under the curve

FIGO Fédération Internationale de Gynécologie Obstétrique (International Federation of Gynecology and Obstetrics)

$\mathrm{G}$ [1-3] grading
} 


\section{References}

[1] R. Angioli, S. Capriglione, A. Aloisi, D. Luvero, E.V. Cafa, N. Dugo, R. Montera, C. De Cicco Nardone, C. Terranova and F. Plotti, REM (risk of endometrial malignancy): A proposal for a new scoring system to evaluate risk of endometrial malignancy, Clin Cancer Res 19 (2013), 5733-5739.

[2] R. Angioli, S. Capriglione, G. Scaletta, A. Aloisi, A. Miranda, C. De Cicco Nardone, C. Terranova and F. Plotti, The role of HE4 in endometrial cancer recurrence: how to choose the optimal follow-up program, Tumour Biol 37 (2016), 49734978.

[3] R. Angioli, A. Miranda, A. Aloisi, R. Montera, S. Capriglione, C. De Cicco Nardone, C. Terranova and F. Plotti, A critical review on HE4 performance in endometrial cancer: Where are we now? Tumour Biol 35 (2014), 881-887.

[4] R. Angioli, F. Plotti, S. Capriglione, R. Montera, P. Damiani, R. Ricciardi, A. Aloisi, D. Luvero, E.V. Cafa, N. Dugo, M. Angelucci and P. Benedetti-Panici, The role of novel biomarker HE4 in endometrial cancer: A case control prospective study, Tumour Biol 34 (2013), 571-576.

[5] R. Angioli, F. Plotti, S. Capriglione, G. Scaletta, N. Dugo, A Aloisi, C.L. Piccolo, R. Del Vescovo, C. Terranova and B.B. Zobel, Preoperative local staging of endometrial cancer: The challenge of imaging techniques and serum biomarkers, Arch Gynecol Obstet 294 (2016), 1291-1298.

[6] M. Bauer, J.C. Eickhoff, M.N. Gould, C. Mundhenke, N. Maass and A. Friedl, Neutrophil gelatinase-associated lipocalin (NGAL) is a predictor of poor prognosis in human primary breast cancer, Breast Cancer Res Treat 108 (2008), 389-397.

[7] D. Bolignano, V. Donato, A. Lacquaniti, M.R. Fazio, C. Bono, G. Coppolino and M. Buemi, Neutrophil gelatinaseassociated lipocalin (NGAL) in human neoplasias: A new protein enters the scene, Cancer Lett 288 (2010), 10-16.

[8] A. Booth, A. Magnuson, J. Fouts and M.T. Foster, Adipose tissue: An endocrine organ playing a role in metabolic regulation, Horm Mol Biol Clin Investig 26 (2016), 25-42.

[9] B. Cabia, S. Andrade, M.C. Carreira, F.F. Casanueva and A.B. Crujeiras, A role for novel adipose tissue-secreted factors in obesity-related carcinogenesis, Obes Rev 17 (2016), 361-376.

[10] S. Candido, R. Maestro, J. Polesel, A. Catania, F. Maira, S.S Signorelli, J.A. McCubrey and M. Libra, Roles of neutrophil gelatinase-associated lipocalin (NGAL) in human cancer, Oncotarget 5 (2014), 1576-1594.

[11] H. Cho and J.H. Kim, Lipocalin2 expressions correlate significantly with tumor differentiation in epithelial ovarian cancer, J Histochem Cytochem 57 (2009), 513-521.

[12] E.R. DeLong, D.M. DeLong and D.L. Clarke-Pearson, Comparing the areas under two or more correlated receiver operating characteristic curves: A nonparametric approach, Biometrics 44 (1988), 837-845.

[13] Z.P. Du, Z. Lv, B.L. Wu, Z.Y. Wu, J.H. Shen, J.Y. Wu, X.E. $\mathrm{Xu}$, Q. Huang, J. Shen, H.B. Chen, E.M. Li and L.Y. Xu, Neutrophil gelatinase-associated lipocalin and its receptor: Independent prognostic factors of oesophageal squamous cell carcinoma, J Clin Pathol 64 (2011), 69-74.

[14] Z.P. Du, H.M. Yuan, B.L. Wu, J.X. Chang, Z. Lv, J. Shen, J.Y. Wu, H.B. Chen, E.M. Li and L.Y. Xu, Neutrophil gelatinaseassociated lipocalin in gastric carcinoma cells and its induction by TPA are controlled by C/EBPbeta, Biochem Cell Biol 89 (2011), 314-324.

[15] E. Gasiorowska, M. Magnowska, N. Izycka, W. Warchol and E. Nowak-Markwitz, The role of HE4 in differentiating be- nign and malignant endometrial pathology, Ginekol Pol 87 (2016), 260-264.

[16] L. Hu, S. Du, W. Guo, D. Chen and Y. Li, Comparison of serum human epididymis protein 4 and carbohydrate antigen 125 as markers in endometrial cancer: A meta-analysis, Int $J$ Gynecol Cancer 26 (2016), 331-340.

[17] L. Hu, W. Hittelman, T. Lu, P. Ji, R. Arlinghaus, I. Shmulevich, S.R. Hamilton and W. Zhang, NGAL decreases Ecadherin-mediated cell-cell adhesion and increases cell motility and invasion through Rac1 in colon carcinoma cells, Lab Invest 89 (2009), 531-548.

[18] L. Kjeldsen, C. Koch, K. Arnljots and N. Borregaard, Characterization of two ELISAs for NGAL, a newly described lipocalin in human neutrophils, J Immunol Methods 198 (1996), 155-164.

[19] X. Leng, T. Ding, H. Lin, Y. Wang, L. Hu, J. Hu, B. Feig, W. Zhang, L. Pusztai, W.F. Symmans, Y. Wu and R.B. Arlinghaus, Inhibition of lipocalin 2 impairs breast tumorigenesis and metastasis, Cancer Res 69 (2009), 8579-8584.

[20] X. Leng, H. Lin, T. Ding, Y. Wang, Y. Wu, S. Klumpp, T. Sun, Y. Zhou, P. Monaco, J. Belmont, A. Aderem, S. Akira, R. Strong and R. Arlinghaus, Lipocalin 2 is required for BCR-ABL-induced tumorigenesis, Oncogene 27 (2008), 6110-6119.

[21] C.J. Liao, Y.H. Huang, H.K. Au, L.M. Wang and S.T. Chu, The cancer marker neutrophil gelatinase-associated lipocalin is highly expressed in human endometrial hyperplasia, $M o l$ Biol Rep 39 (2012), 1029-1036.

[22] R. Lim, N. Ahmed, N. Borregaard, C. Riley, R. Wafai, E.W. Thompson, M.A. Quinn and G.E. Rice, Neutrophil gelatinase-associated lipocalin (NGAL) an early-screening biomarker for ovarian cancer: NGAL is associated with epidermal growth factor-induced epithelio-mesenchymal transition, Int J Cancer 120 (2007), 2426-2434.

[23] H.H. Lin, C.J. Liao, Y.C. Lee, K.H. Hu, H.W. Meng and S.T. Chu, Lipocalin-2-induced cytokine production enhances endometrial carcinoma cell survival and migration, Int J Biol Sci 7 (2011), 74-86.

[24] G. Lippi, T. Meschi, A. Nouvenne, C. Mattiuzzi and L. Borghi, Neutrophil gelatinase-associated lipocalin in cancer, Adv Clin Chem 64 (2014), 179-219.

[25] X. Liu, F. Zhao, L. Hu and Y. Sun, Value of detection of serum human epididymis secretory protein 4 and carbohydrate antigen 125 in diagnosis of early endometrial cancer of different pathological subtypes, Onco Targets Ther 8 (2015), 12391243.

[26] M. Mannelqvist, I.M. Stefansson, E. Wik, K. Kusonmano, M.B. Raeder, A.M. Oyan, K.H. Kalland, M.A. Moses, H.B. Salvesen and L.A. Akslen, Lipocalin 2 expression is associated with aggressive features of endometrial cancer, $B M C$ Cancer 12 (2012), 169.

[27] T. Miyamoto, R. Asaka, A. Suzuki, A. Takatsu, H. Kashima and T. Shiozawa, Immunohistochemical detection of a specific receptor for lipocalin2 (solute carrier family 22 member 17, SLC22A17) and its prognostic significance in endometrial carcinoma, Exp Mol Pathol 91 (2011), 563-568.

[28] T. Miyamoto, H. Kashima, A. Suzuki, N. Kikuchi, I. Konishi, N. Seki and T. Shiozawa, Laser-captured microdissectionmicroarray analysis of the genes involved in endometrial carcinogenesis: Stepwise up-regulation of lipocalin2 expression in normal and neoplastic endometria and its functional relevance, Hum Pathol 42 (2011), 1265-1274.

[29] N. Moniaux, S. Chakraborty, M. Yalniz, J. Gonzalez, V.K. Shostrom, J. Standop, S.M. Lele, M. Ouellette, P.M. Pour, 
A.R. Sasson, R.E. Brand, M.A. Hollingsworth, M. Jain and S.K. Batra, Early diagnosis of pancreatic cancer: Neutrophil gelatinase-associated lipocalin as a marker of pancreatic intraepithelial neoplasia, Br J Cancer 98 (2008), 1540-1547.

[30] K. Paine and D.R. Flower, The lipocalin website, Biochim Biophys Acta 1482 (2000), 351-352.

[31] B. Patsner and G.W. Yim, Predictive value of preoperative serum CA-125 levels in patients with uterine cancer: The Asian experience 2000 to 2012, Obstet Gynecol Sci 56 (2013), 281-288.

[32] F. Plotti, S. Capriglione, G. Scaletta, D. Luvero, S. Lopez, F.F. Nastro, C. Terranova, C. De Cicco Nardone, R. Montera and R. Angioli, Implementing the Risk of Endometrial Malignancy Algorithm (REM) adding obesity as a predictive factor: Results of REM-B in a single-center survey, Eur J Obstet Gynecol Reprod Biol 225 (2018), 51-56.

[33] F. Plotti, S. Capriglione, C. Terranova, R. Montera, A. Aloisi, P. Damiani, L. Muzii, G. Scaletta, P. Benedetti-Panici and R. Angioli, Does HE4 have a role as biomarker in the recurrence of ovarian cancer?, Tumour Biol 33 (2012), 2117-2123.

[34] G. Scambia, A. Gadducci, P.B. Panici, E. Foti, M. Ferdeghini, G. Ferrandina, M. Amoroso, C. Castellani, V. Facchini and S Mancuso, Combined use of CA 125 and CA 15-3 in patients with endometrial carcinoma, Gynecol Oncol 54 (1994), 292297.

[35] N. Takeshima, Y. Hirai, K. Yamauchi and K. Hasumi, Clinical usefulness of endometrial aspiration cytology and CA-125 in the detection of fallopian tube carcinoma, Acta Cytol 41 (1997), 1445-1450.

[36] H.J. Wang, X.J. He, Y.Y. Ma, X.T. Jiang, Y.J. Xia, Z.Y. Ye, Z.S. Zhao and H.Q. Tao, Expressions of neutrophil gelatinaseassociated lipocalin in gastric cancer: A potential biomarker for prognosis and an ancillary diagnostic test, Anat Rec (Hoboken) 293 (2010), 1855-1863.

[37] L. Wang, H. Li, J. Wang, W. Gao, Y. Lin, W. Jin, G. Chang, R. Wang, Q. Li, L. Ma and T. Pang, C/EBP zeta targets to neutrophil gelatinase-associated lipocalin (NGAL) as a repressor for metastasis of MDA-MB-231 cells, Biochim Biophys Acta 1813 (2011), 1803-1813.
[38] Y. Wang, K.S. Lam, E.W. Kraegen, G. Sweeney, J. Zhang, A.W. Tso, W.S. Chow, N.M. Wat, J.Y. Xu, R.L. Hoo and A. $\mathrm{Xu}$, Lipocalin-2 is an inflammatory marker closely associated with obesity, insulin resistance, and hyperglycemia in humans, Clin Chem 53 (2007), 34-41.

[39] Y. Wang and T. Zeng, Neutrophil gelatinase-associated lipocalin protein as a biomarker in the diagnosis of breast cancer: A meta-analysis, Biomed Rep 1 (2013), 479-483.

[40] Y. Wang and T.T. Zeng, Clinical significance of neutrophil gelatinase-associated lipocalin (NGAL) in colorectal cancer: A meta-analysis, Genet Mol Res 13 (2014), 7102-7112.

[41] Q.W. Yan, Q. Yang, N. Mody, T.E. Graham, C.H. Hsu, Z. $\mathrm{Xu}$, N.E. Houstis, B.B. Kahn and E.D. Rosen, The adipokine lipocalin 2 is regulated by obesity and promotes insulin resistance, Diabetes 56 (2007), 2533-2540.

[42] J. Yang and M.A. Moses, Lipocalin 2: A multifaceted modulator of human cancer, Cell Cycle 8 (2009), 2347-2352.

[43] H. Zhang, L. Xu, D. Xiao, J. Xie, H. Zeng, Z. Wang, X. Zhang, Y. Niu, Z. Shen, J. Shen, X. Wu and E. Li, Upregulation of neutrophil gelatinase-associated lipocalin in oesophageal squamous cell carcinoma: Significant correlation with cell differentiation and tumour invasion, J Clin Pathol 60 (2007), 555-561.

[44] J. Zhang, Y. Wu, Y. Zhang, D. Leroith, D.A. Bernlohr and X. Chen, The role of lipocalin 2 in the regulation of inflammation in adipocytes and macrophages, Mol Endocrinol 22 (2008), 1416-1426.

[45] X.F. Zhang, Y. Zhang, X.H. Zhang, S.M. Zhou, G.G. Yang, O.C. Wang, G.L. Guo, G.Y. Yang and X.Q. Hu, Clinical significance of Neutrophil gelatinase-associated lipocalin (NGAL) expression in primary rectal cancer, BMC Cancer 9 (2009), 134. 